\title{
Thermoelectric properties of the misfit cobaltate $\mathrm{Ca}_{3} \mathrm{Co}_{4} \mathrm{O}_{9}$
}

\author{
Bin Amin, ${ }^{1}$ Ulrich Eckern, ${ }^{2, a)}$ and Udo Schwingenschlögl ${ }^{1, b)}$ \\ ${ }^{1}$ King Abdullah University of Science and Technology (KAUST), Physical Science and Engineering Division \\ (PSE), Thuwal 23955-6900, Saudi Arabia \\ ${ }^{2}$ University of Augsburg, Institute of Physics, 86135 Augsburg, Germany
}

(Received 6 April 2017; accepted 23 May 2017; published online 9 June 2017)

\begin{abstract}
The layered misfit cobaltate $\mathrm{Ca}_{3} \mathrm{Co}_{4} \mathrm{O}_{9}$, also known as $\mathrm{Ca}_{2} \mathrm{CoO}_{3}\left[\mathrm{CoO}_{2}\right]_{1.62}$, is a promising p-type thermoelectric oxide. Employing density functional theory, we study its electronic structure and determine, on the basis of Boltzmann theory within the constant-relaxation-time approximation, the thermoelectric transport coefficients. The dependence on strain and temperature is determined. In particular, we find that the $x x$-component of the thermopower is strongly enhanced, while the $y y$ component is strongly reduced, when applying $2 \%$ tensile strain. A similar anisotropy is also found in the power factor. The temperature dependence of the conductivity in the $a-b$ plane is found to be rather weak above $200 \mathrm{~K}$, which clearly indicates that the experimentally observed transport properties are dominated by inhomogeneities arising during sample growth, i.e., they are not intrinsic. Published by AIP Publishing. [http://dx.doi.org/10.1063/1.4984960]
\end{abstract}

Since the discovery of a large thermoelectric power in the layered cobaltate $\mathrm{NaCoO}_{2}$ two decades ago, ${ }^{1}$ this system and closely related ones, particularly $\mathrm{Ca}_{3} \mathrm{Co}_{4} \mathrm{O}_{9}$ (CCO), have been studied intensively, both experimentally ${ }^{2-26}$ and theoretically; ${ }^{27-32}$ see also Refs. 33-35 for recent reviews. Layered cobalt oxides such as $\mathrm{CCO}$ are of interest because of the close proximity of the rock-salt $\mathrm{Ca}_{2} \mathrm{CoO}_{3}$ and hexagonal $\mathrm{CoO}_{2}$ subsystems, which results in a high Seebeck coefficient, low thermal conductivity, and, hence, high figure of merit at elevated temperature.

For example, Wakisaka et al. ${ }^{13}$ studied the electronic structure by x-ray photoemission spectroscopy, ultraviolet photoemission spectroscopy, and unrestricted Hartree-Fock calculations. They derived a charge-transfer energy of $1 \mathrm{eV}$ between the $\mathrm{Co}$ sites in the $\mathrm{Ca}_{2} \mathrm{CoO}_{3}$ and $\mathrm{CoO}_{2}$ subsystems and a $d$ - $d$ Coulomb interaction energy of $6.5 \mathrm{eV}$. Huang et $a l .{ }^{18}$ investigated the structural, electrical, and thermal transport properties of $\mathrm{CCO}$ ceramics sintered in a high magnetic field, arguing that this technique was efficient for enhancing the thermopower. High-resolution photoemission spectroscopy was used by Takeuchi et al. ${ }^{6,7}$ to investigate the electronic structure. These authors argued that the high thermopower was due to the metallic conduction in the hexagonal $\mathrm{CoO}_{2}$ layers. According to Refs. 3 and 14, the rocksalt subsystem mainly shortens the phonon mean free path and thus lowers the thermal conductivity, while the hexagonal subsystem is responsible for the electrical conductivity. In addition, the magneto-transport properties are remarkable; ${ }^{2,10,17}$ in particular, a high-temperature (above $600 \mathrm{~K}$ ) spin state transition has been discovered very recently. ${ }^{36,37}$

First principles calculations were performed by Asahi et $a l .{ }^{27}$ using a $3 / 2$ ratio for the $b_{1}$ (rock-salt) and $b_{2}\left(\mathrm{CoO}_{2}\right)$ lattice parameters, $\left(b_{1} / b_{2}\right)_{\exp }=1.62$, arguing that the optimized structure with distorted octahedra was in agreement with the experiment. The authors reported on p-type conductivity in the rock-salt subsystem, where the Fermi energy lies

\footnotetext{
${ }^{a}$ Electronic mail: ulrich.eckern@physik.uni-augsburg.de

${ }^{\text {b)} E l e c t r o n i c ~ m a i l: ~ u d o . s c h w i n g e n s c h l o g l @ k a u s t . e d u . s a ~}$
}

in the crystal-field gap of the $3 d$ states of the $\mathrm{CoO}_{2}$ subsystem. Tyson et al. ${ }^{15}$ combined temperature-dependent local structure measurements with first-principles calculations to develop a three-dimensional structure model of misfit $\mathrm{Ca}_{3} \mathrm{Co}_{4} \mathrm{O}_{9}$. They found a low coordination of $\mathrm{Co}$ in the rock-salt layer, which is due to the formation of Co chains in the $a-b$ plane linked to the $\mathrm{CaO}$ layers by $\mathrm{Co}-\mathrm{O}$ bonds oriented along the $c$ axis, enabling high electrical and low thermal conductivities.

Combining experiment, ab-initio calculations, and the semiclassical Boltzmann transport theory to investigate the anisotropy of the thermopower in $\mathrm{Ca}_{3} \mathrm{Co}_{4} \mathrm{O}_{9}$ single crystals, Tang et $a l .{ }^{17}$ found a strongly anisotropic topology of the Fermi surface. Rebola et $a .^{29}$ investigated the atomic and electronic structures employing density functional theory (DFT) and its extension $(\mathrm{DFT}+U)$. Both methods led to good agreement of the structural parameters with the experiment.

In this work, we consider the 5/3 rational approximation, optimize the structure employing density functional theory (in the VASP implementation), and determine the thermoelectric transport coefficients on the basis of BoltzTrap. The transport properties are calculated for the pristine and for strained systems, as a function of temperature. Substrate effects are discussed for a specific example $\left(\mathrm{SrTiO}_{3}\right)$.

As mentioned above, the monoclinic structure of misfit $\mathrm{Ca}_{3} \mathrm{Co}_{4} \mathrm{O}_{9}$ is built of rock-salt $\mathrm{Ca}_{2} \mathrm{CoO}_{3}$ sandwiched between hexagonal $\mathrm{CoO}_{2}$ along the $c$ direction. The subsystems share the $a$ and $c$ lattice parameters. The $b$ lattice parameters differ so that a rational approximation with a minimal lattice mismatch has to be chosen. Asahi et al. ${ }^{27}$ used $b_{1} / b_{2}=3 / 2$ corresponding to a mismatch of $7 \%$, whereas the $5 / 3$ ratio employed by Rebola et al. $^{29}$ reduces the mismatch to $3 \%$. In contrast to the experiment, ${ }^{6,7}$ the $3 / 2$ ratio results in $3 d$ states of the $\mathrm{Ca}_{2} \mathrm{CoO}_{3}$ subsystem at the Fermi energy, while the $5 / 3$ ratio leads only to minor contributions. Thus, we consider $b_{1} / b_{2}=5 / 3$.

The $a$ and $c$ lattice parameters are initially set to the experimental values, whereas for the $b$ lattice parameter, a value of $5 \times 2.82 \AA=14.1 \AA$ (taken from the $\mathrm{CoO}_{2}$ subsystem) is used. Then, the supercell is fully optimized, with an on-site Coulomb interaction of $5 \mathrm{eV}$. The unrelaxed and 
relaxed structures are presented in Fig. 1. The optimized lattice parameters and the corresponding bond lengths are summarized in Table I that also includes a comparison to experimental data. The optimized $b$ lattice parameter is found to be determined by the $\mathrm{CoO}_{2}$ subsystem $\left(b_{2}\right)$, whereas the $\mathrm{Ca}_{2} \mathrm{CoO}_{3}$ subsystem $\left(b_{1}\right)$ turns out to be intrinsically strained. Figure 1 indeed shows strong distortions in the rock-salt subsystem.

For the structural optimization, we use the projector augmented wave method as implemented in the VASP code ${ }^{38}$ relaxing both the atomic positions and lattice parameters. A plane-wave cutoff energy of $530 \mathrm{eV}$ and a residual force criterion of $0.02 \mathrm{eV} / \AA$ are used together with a Monkhorst-Pack $6 \times 2 \times 3 \mathrm{k}$-mesh. The generalized gradient approximation in the Perdew-Burke-Ernzerhof flavor $^{39}$ is employed, and spin-polarization is taken into account.

In order to model the electronic correlations affecting the localized Co $d$ states, on-site Coulomb repulsions of $U=3 \mathrm{eV}$ and $5 \mathrm{eV}$ (with $J=1 \mathrm{eV}$ ) have been tested. For $U=5 \mathrm{eV}$, the density of states is found to be in good agreement with the experiment, ${ }^{6,7}$ whereas for $U=3 \mathrm{eV}$, states of the $\mathrm{Ca}_{2} \mathrm{CoO}_{3}$ subsystem appear at the Fermi energy. It turns out that the self-consistent solution depends critically on the initial value of the Co magnetic moment. The values of 1 , 1.5 , and $2 \mu_{B}$ have been compared. In the former $\left(1 \mu_{B}\right)$ case, no substantial magnetic moments are formed in the $\mathrm{CoO}_{2}$ subsystem, and the total energy is strongly reduced. Thus, only this case is further addressed. Both $2 \%$ and $4 \%$ compressive volumetric strain and $2 \%$ tensile volumetric strain are studied, for which the atomic positions are re-relaxed.

Precise electronic structures are obtained by the Wien $2 \mathrm{k}^{40}$ code using $U=5 \mathrm{eV}$ and $J=1 \mathrm{eV}$. The parameter $R_{\mathrm{mt}} K_{\max }$ is set to 8 , with $G_{\max }=24$, and 320 k-points are considered in the irreducible wedge of the Brillouin zone. The transport properties are calculated using semiclassical Boltzmann theory, as implemented in the BoltzTraP code, ${ }^{41,42}$ employing $2000 \mathrm{k}$-points in the irreducible wedge of the Brillouin zone.

We expect this procedure of calculating the transport coefficients to be applicable in the intermediate metallic regime; hence, we show the results only for $T$ larger than $200 \mathrm{~K}$. Furthermore, within the present approach, neither the structural change near $400 \mathrm{~K}$ (Ref. 20) nor the spin state transition above $600 \mathrm{~K}$ (Refs. 36 and 37) is covered.

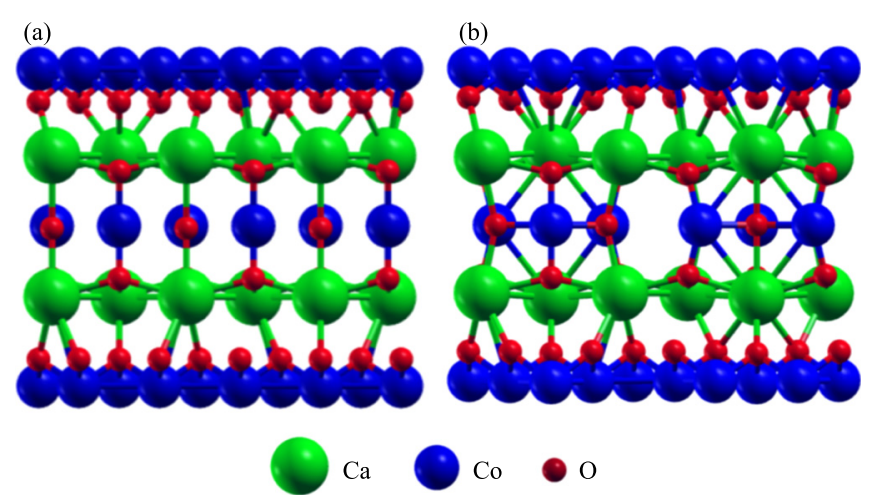

FIG. 1. Structure (a) before and (b) after relaxation (5/3 ratio of $\mathrm{CoO}_{2}$ and $\left.\mathrm{Ca}_{2} \mathrm{CoO}_{3}\right)$.
TABLE I. Calculated and experimental structure parameters. All lengths are given in units of $\AA$, and the angle $\beta$ in degrees.

\begin{tabular}{lccccccccc}
\hline \hline & $a$ & $b_{1}$ & $b_{2}$ & $c$ & $\beta$ & $\mathrm{Ca}-\mathrm{O}$ & $\mathrm{Co}_{1}-\mathrm{O}_{1}$ & $\mathrm{Co}_{1}-\mathrm{O}_{2}$ & $\mathrm{Co}_{2}-\mathrm{O}$ \\
\hline GGA & 4.89 & 4.70 & 2.82 & 10.73 & 98.4 & 2.46 & 2.00 & 1.82 & 1.92 \\
GGA+U & 4.88 & 4.71 & 2.82 & 10.71 & 98.4 & 2.46 & 2.01 & 1.81 & 1.92 \\
Exp. (Ref. 29) & 4.83 & 4.56 & 2.82 & 10.84 & 98.1 & 2.40 & 2.05 & 2.30 & 1.92 \\
\hline \hline
\end{tabular}

The density of states in the top row of Fig. 2(a) shows that only the spin up Co $3 d$ states of the $\mathrm{CoO}_{2}$ subsystem contribute at the Fermi energy, in agreement with the experiment. ${ }^{6,7}$ According to the orbitally projected densities of states, these contributions are mainly due to the $t_{2 g}\left(d_{x y}, d_{x z}\right.$, and $d_{y z}$ ) orbitals. Figure 2(a) qualitatively agrees with the findings of Ref. 29; minor deviations can be attributed to different computational schemes employed.

The thermoelectric properties are addressed in Fig. 3 where the left, middle, and right columns refer to the $x x, y y$, and $z z$ components, respectively. A comparison of the calculated electrical conductivity at $300 \mathrm{~K}$ with the experiment in Ref. 4 ( $\rho \simeq 10.7 \mathrm{~m} \Omega \mathrm{cm}$, where $\rho$ denotes the resistivity) leads to a relaxation time of $3 \times 10^{-16} \mathrm{~s}$, which is used in the following. We note that the conductivity is enhanced in the $a-b$ plane (average of the $x x$ and $y y$ components). A maximal conductivity is achieved around $400 \mathrm{~K}$. The positive values of the Seebeck coefficient reflect a hole character of the majority charge carriers. In the $a-b$ plane, the Seebeck coefficient increases with temperature and starts saturating around $400 \mathrm{~K}$, which agrees well with the experiment, ${ }^{2}$ whereas the $z z$ component does not saturate. At $300 \mathrm{~K}$, we find for the total Seebeck coefficient (average of the $x x, y y$, and $z z$ components) a value close to $60 \mu \mathrm{V} / \mathrm{K}$, whereas in Refs. 27 and 29 , values of $47 \mu \mathrm{V} / \mathrm{K}$ and $227 \mu \mathrm{V} / \mathrm{K}$, respectively, were predicted, and measurements result in $135 \mu \mathrm{V} / \mathrm{K}^{2,8,19}$ The power factor largely resembles the temperature dependence of the Seebeck coefficient.

The densities of states obtained for strained $\mathrm{CCO}$ are also shown in Fig. 2, for 2\% tensile strain, part (b), and 2\% compressive volumetric strain, part (c). In both cases, similar to the unstrained system, only the spin up Co $3 d$ states of the $\mathrm{CoO}_{2}$ subsystem contribute at the Fermi energy, while the $\mathrm{Ca}_{2} \mathrm{CoO}_{3}$ subsystem gives essentially no contributions at all. The same applies to the case of $4 \%$ compressive volumetric strain (not shown). The strained systems reproduce the gross features of the unstrained system, except for a shift of the spin down conduction band towards the Fermi energy for increasing compressive strain.

The electrical conductivity increases slightly with increasing compressive strain (see Fig. 3). The overall behaviors of the Seebeck coefficient and power factor as functions of the temperature do not change under compressive strain, but the values clearly decrease, particularly for the $a-b$ plane. Interestingly, under tensile strain, the electrical conductivity decreases significantly, because of an energetic shift and enhanced localization of the Co $3 d$ states of the $\mathrm{CoO}_{2}$ subsystem at the Fermi energy (see Fig. 2(b)), while the in-plane Seebeck coefficient increases at high temperature. The total Seebeck coefficient increases at high temperature more strongly than under compressive strain but less than 

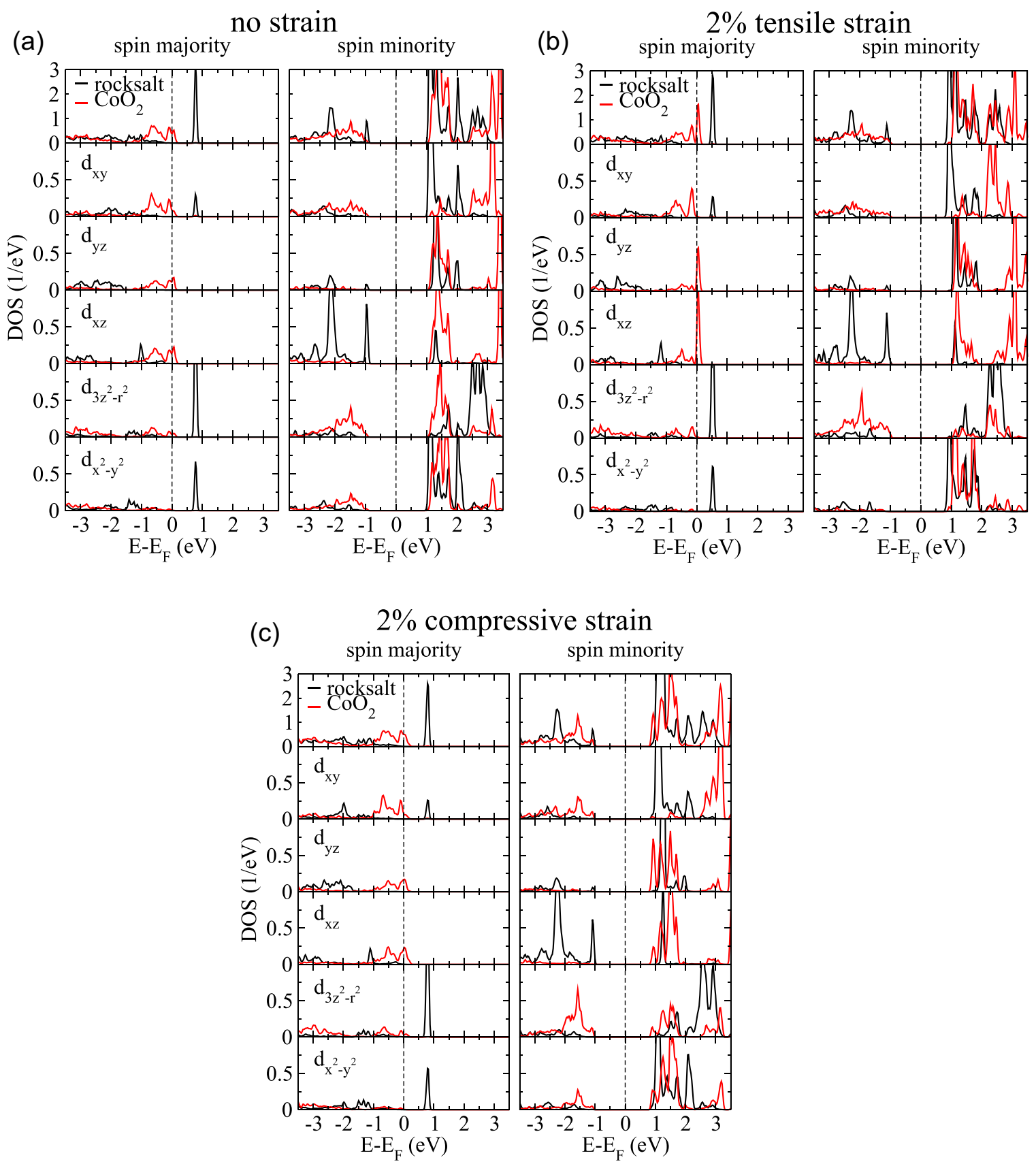

FIG. 2. Spin majority and minority densities of states without strain (a), under $2 \%$ tensile volumetric strain (b), and under $2 \%$ compressive volumetric strain (c). The DOS for $4 \%$ compressive strain is found to be almost indistinguishable from the latter. In correspondence with the behavior of the DOS, we do not find any qualitative change of the crystal structure under strain.

for the unstrained case. However, the decrease in the electrical conductivity leads to a decrease in the power factor under tensile strain. A strong anisotropy in the Seebeck coefficient in the $a-b$ plane is apparent. The overall behavior of the thermal conductivity as function of strain is similar to the conductivity, except that $\kappa_{e}$ increases approximately linearly in $T$.

It has been demonstrated for various substrates that first a rocksalt $\mathrm{Ca}_{2} \mathrm{CoO}_{3}$ buffer layer of up to $25 \mathrm{~nm}$ thickness is formed before the growth of $\mathrm{Ca}_{3} \mathrm{Co}_{4} \mathrm{O}_{9}$ sets in. ${ }^{19}$ The effect is stronger on (001)-oriented perovskite substrates than on hexagonal sapphire substrates, where the buffer layer extends only over a few nm. Next to the buffer layer, still many $\mathrm{CoO}_{2}$ stacking faults are observed, which are assumed to enhance the phonon scattering and thus the Seebeck coefficient in films up to $50 \mathrm{~nm}$ thickness. ${ }^{19}$ Due to the presence of the buffer layer, it seems unlikely that the substrate has a strong effect on $\mathrm{Ca}_{3} \mathrm{Co}_{4} \mathrm{O}_{9}$. This point of view is also supported by the lattice parameters obtained from high-resolution electron microscopy. ${ }^{8}$ However, in order to ascertain whether in-plane strain from the substrate could have any positive effect on the thermoelectric performance, we study in the following a representative case, $\mathrm{SrTiO}_{3}$, explicitly.

To this end, we strain the $\mathrm{Ca}_{3} \mathrm{Co}_{4} \mathrm{O}_{9}$ supercell by forcing both the $a$ and $b$ lattice parameters (original values $a=4.89 \AA$ and $b=14.1 \AA$ ) to the lattice parameter of $\mathrm{SrTiO}_{3}$ (3.905 $\AA$ and $3 \times 3.905 \AA$ ). This corresponds to rather strong compressions of $20 \%$ for $a$ and $17 \%$ for $b$. We then optimize the $c$ lattice parameter relaxing the atomic positions (see Fig. 4) and obtain an expansion of $16 \%$ to $12.45 \AA$. Due to the strong distortions, the densities of states in Fig. 5 show 

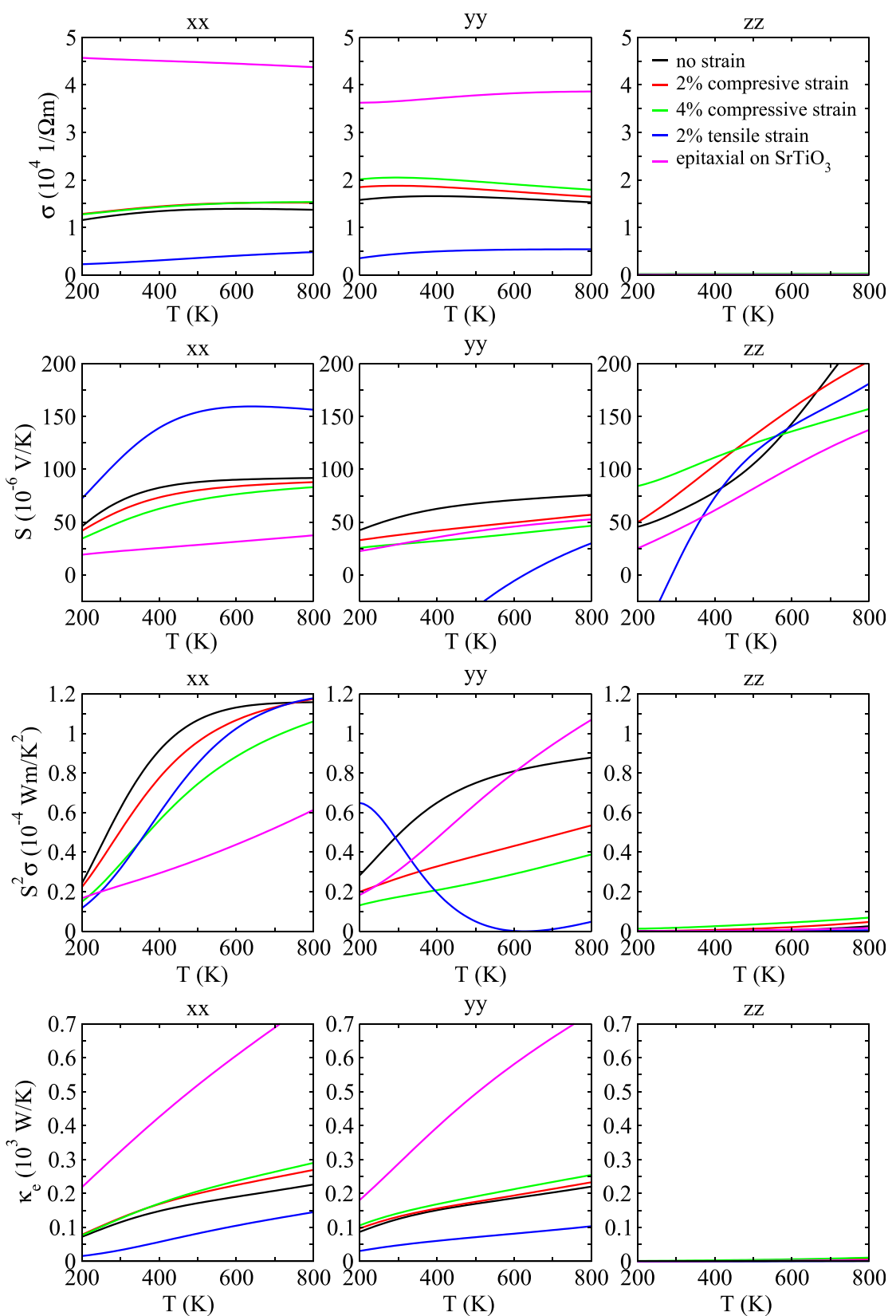

significant differences compared to Fig. 2(c). The consequences for the thermoelectric properties are demonstrated in Fig. 3 as pink lines. As expected from the strong compression, the electrical and thermal conductivities increase, whereas the Seebeck coefficient and the power factor show no improvement over the unstrained material for most temperatures. These results indicate that $\mathrm{Ca}_{3} \mathrm{Co}_{4} \mathrm{O}_{9}$ samples are not intrinsically strained and that such strain even would not be fruitful. Although it is computationally very demanding, a simulation of stacking faults could give further insights.

In summary, we have determined, on the basis of a DFT $+U$ calculation that predicts a metallic ground state for the misfit cobaltate $\mathrm{Ca}_{3} \mathrm{Co}_{4} \mathrm{O}_{9}$, the temperature dependence of the thermoelectric transport coefficients in the constantrelaxation-time approximation. We studied the pristine system (i.e., the 5/3 rational approximation) and its behavior under tensile and compressive strain. The results are expected to be applicable for not too low temperature, $T \geqslant 200 \mathrm{~K}$. In order to explain the measured room-temperature resistivity, ${ }^{4}$ we have chosen the relaxation time to be given by $3 \times 10^{-16} \mathrm{~s}$, slightly

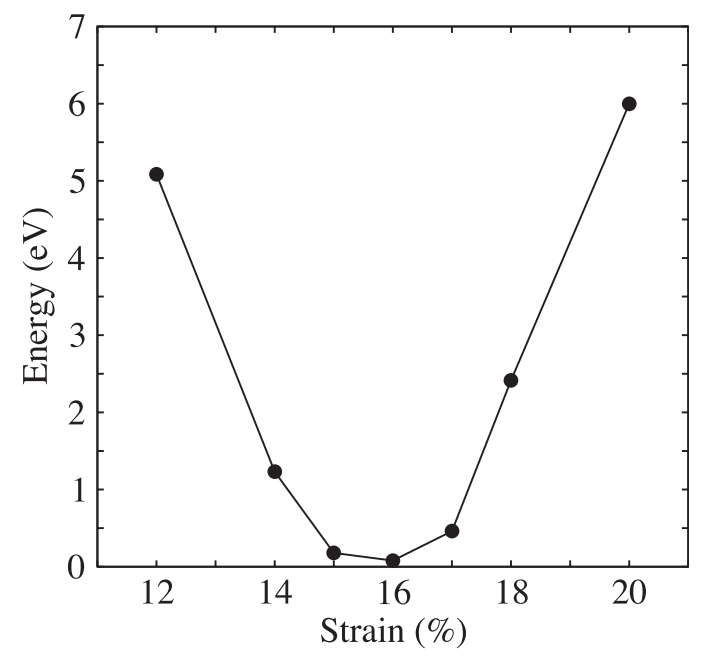

FIG. 4. Optimization of the $c$ lattice parameter assuming $20 \%$ and $17 \%$ compression, respectively, for the $a$ and $b$ lattice parameters.
FIG. 3. Electrical conductivity $(\sigma)$, Seebeck coefficient $(S)$, power factor $\left(S^{2} \sigma\right)$, and electronic part of the thermal conductivity $\left(\kappa_{e}\right)$, for $\tau=3$ $\times 10^{-16} \mathrm{~s}$. The conductivity can be related to an effective carrier density, $n_{\text {eff }}=\sigma \times m / e^{2} \tau$; using for $m$ the electron rest mass, this quantity is given by $1.2 \times 10^{21} \mathrm{~cm}^{-3}$ for $\sigma=1 \times 10^{4}$ $(\Omega \mathrm{m})^{-1}$. Thus, the results presented in the topmost row also give some indication of the strain dependence of the relevant carrier density. 
epitaxial on $\mathrm{SrTiO}_{3}$ spin majority

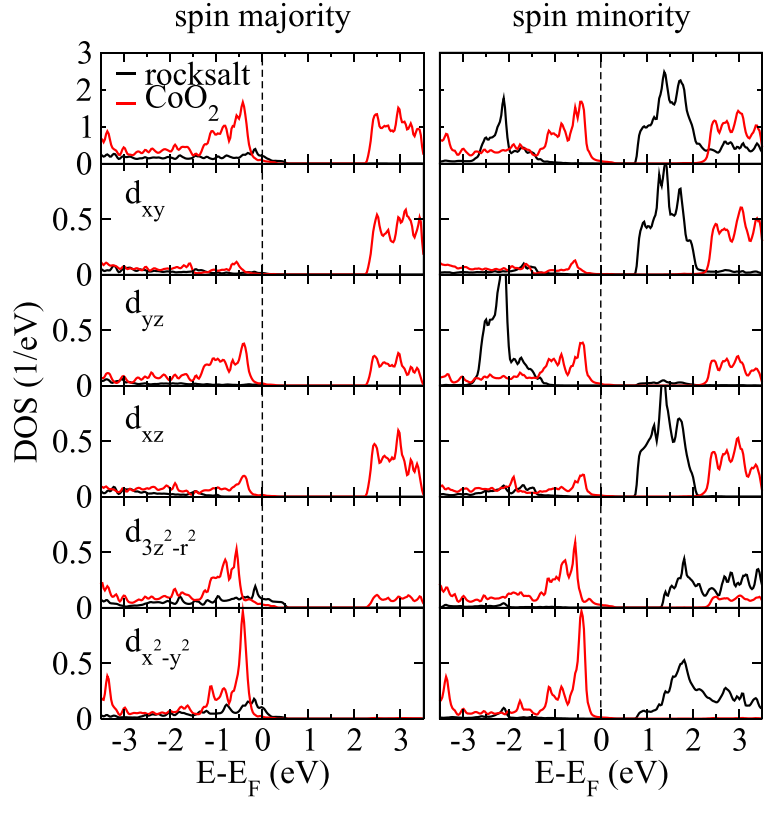

FIG. 5. Spin majority and minority densities of states when matching the in-plane lattice parameters to $\mathrm{SrTiO}_{3}$.

smaller than the value used in Ref. $32,8 \times 10^{-16}$ s. Expressed in a different way, we find $\sigma / \tau$ to be given by $\simeq 0.37 \times 10^{15}$ $(\mathrm{m} \Omega \mathrm{cm} \mathrm{s})^{-1}$, which is by about a factor of four larger than the corresponding value of Lemal et al. ${ }^{32}$ Note, however, that the reported resistivities vary considerably, ranging from $\simeq 2$ for single crystals $^{5}$ to $\simeq 170 \mathrm{~m} \Omega \mathrm{cm}$ for thin films fabricated on an $\mathrm{LaAlO}_{3}$ substrate using the pulsed laser deposition (PLD) technique. ${ }^{22}$ Another group, also using PLD but on (001)-silicon with a thin epitaxial yttria-stabilized zirconia (YSZ) buffer layer, reported $\simeq 2-13 \mathrm{~m} \Omega \mathrm{cm}$, depending on the microstructure. $^{25}$ Clearly, these results indicate a strong dependence of transport properties on the growth conditions and the choice of the substrate. (Note that we discuss in this paragraph only in-plane transport at room temperature.)

Finally, we note that the conductivities found in CCO are considerably smaller than those which are observed for other layered compounds, e.g., the delafossites $\mathrm{PdCoO}_{2}$ and $\mathrm{PtCoO}_{2},{ }^{43,44}$ which might raise questions concerning the applicability of the Boltzmann transport theory and/or the constant-relaxation-time approximation. In this context, we also recall the simple fact that $\tau \lesssim 10^{-15} \mathrm{~s}$ results in $\hbar / \tau \gtrsim 0.7 \mathrm{eV}$, which, naively speaking, would imply a considerable linewidth, comparable to the relevant energy scales in the density of states discussed above, in the electronic Green's functions. In our opinion, this clearly indicates that not all aspects of transport in CCO are sufficiently well understood. Luckily, the relaxation time cancels in the expression of the thermopower but, even on the Boltzmann equation level, only in the simplest approximation.

We acknowledge helpful discussions with H. Karl and A. Weidenkaff, as well as with M. E. Gruner, R. Pentcheva, and K. I. Wysokiński. This work was financially supported by the German Research Foundation (DFG) through TRR 80. The research reported in this publication was supported by funding from King Abdullah University of Science and Technology (KAUST).
${ }^{1}$ I. Terasaki, Y. Sasago, and K. Uchinokura, Phys. Rev. B 56, R12685(R) (1997).

${ }^{2}$ A. C. Masset, C. Michel, A. Maignan, M. Hervieu, O. Toulemonde, F. Studer, B. Raveau, and J. Hejtmanek, Phys. Rev. B 62, 166 (2000).

${ }^{3}$ S. Lambert, H. Leligny, and D. Grebille, J. Solid State Chem. 160, 322 (2001).

${ }^{4}$ G. Xu, R. Funahashi, M. Shikano, Q. Pu, and B. Liu, Solid State Commun. 124, 73 (2002).

${ }^{5}$ M. Shikano and R. Funahashi, Appl. Phys. Lett. 82, 1851 (2003).

${ }^{6}$ T. Takeuchi, T. Kondo, T. Takami, H. Takahashi, H. Ikuta, U. Mizutani, K. Soda, R. Funahashi, M. Shikano, M. Mikami, S. Tsuda, T. Yokoya, S. Shin, and T. Muro, Phys. Rev. B 69, 125410 (2004).

${ }^{7}$ T. Takeuchi, T. Kondo, K. Soda, U. Mizutani, R. Funahashi, M. Shikano, S. Tsuda, T. Yokoya, S. Shin, and T. Muro, J. Electron Spectrosc. 137-140, 595 (2004).

${ }^{8}$ Y. F. Hu, W. D. Si, E. Sutter, and Q. Li, Appl. Phys. Lett. 86, 082103 (2005).

${ }^{9}$ P. Limelette, V. Hardy, P. Auban-Senzier, D. Jérome, D. Flahaut, S. Hébert, R. Frésard, C. Simon, J. Noudem, and A. Maignan, Phys. Rev. B 71, 233108 (2005).

${ }^{10}$ H. W. Eng, P. Limelette, W. Prellier, C. Simon, and R. Frésard, Phys. Rev. B 73, 033403 (2006).

${ }^{11}$ M. Lee, L. Viciu, L. Li, Y. Wang, M. L. Foo, S. Watauchi, R. A. Pascal, Jr., R. J. Cava, and N. P. Ong, Nat. Mater. 5, 537 (2006).

${ }^{12}$ P. Limelette, S. Hébert, V. Hardy, R. Frésard, C. Simon, and A. Maignan, Phys. Rev. Lett. 97, 046601 (2006).

${ }^{13}$ Y. Wakisaka, S. Hirata, T. Mizokawa, Y. Suzuki, Y. Miyazaki, and T. Kajitani, Phys. Rev. B 78, 235107 (2008).

${ }^{14}$ H. Muguerra, D. Grebille, and F. Bourée, Acta Crystallalogr. B 64, 144 (2008).

${ }^{15}$ T. A. Tyson, Z. Chen, Q. Jie, Q. Li, and J. J. Tu, Phys. Rev. B 79, 024109 (2009).

${ }^{16}$ D. Kenaui, G. Bonnefont, D. Chateigner, G. Fantozzi, M. Gomina, and J. G. Noudem, Mater. Res. Bull. 45, 1240 (2010).

${ }^{17}$ G. D. Tang, H. H. Guo, T. Yang, D. W. Zhang, X. N. Xu, L. Y. Wang, Z. H. Wang, H. H. Wen, Z. D. Zhang, and Y. W. Du, Appl. Phys. Lett. 98, 202109 (2011).

${ }^{18}$ Y. Huang, B. Zhao, J. Fang, R. Ang, and Y. Sun, J. Appl. Phys. 110, 123713 (2011).

${ }^{19}$ Q. Qiao, A. Gulec, T. Paulauskas, S. Kolesnik, B. Dabrowksi, M. Ozdemir, C. Boyraz, D. Mazumdar, A. Gupta, and R. F. Klie, J. Phys.: Condens. Matter 23, 305005 (2011).

${ }^{20}$ T. Wu, T. A. Tyson, H. Chen, J. Bai, H. Wang, and C. Jaye, J. Phys.: Condens. Matter 24, 455602 (2012).

${ }^{21}$ N. Prasoetsopha, S. Pinitsoontorn, and V. Amornkitbamrung, Electron. Mater. Lett. 8, 305 (2012).

${ }^{22}$ P. Jood, G. Peleckis, X. Wang, and S. X. Dou, J. Mater. Res. 28, 1932 (2013).

${ }^{23}$ M. A. Madre, F. M. Costa, N. M. Ferreira, A. Sotelo, M. A. Torres, G. Constantinescu, S. Rasekh, and J. C. Diez, J. Eur. Ceram. Soc. 33, 1747 (2013).

${ }^{24}$ T. Wu, T. A. Tyson, J. Bai, K. Pandya, C. Jaye, and D. Fischer, J. Mater. Chem. C 1, 4114 (2013).

${ }^{25}$ T. Kraus, A. Griesser, O. Klein, M. Fischer, M. Schreck, and H. Karl, Appl. Phys. Lett. 104, 183104 (2014).

${ }^{26}$ Y. Huang, B. Zhao, S. Lin, and Y. Sun, J. Phys. Chem. C 119, 7979 (2015).

${ }^{27}$ R. Asahi, J. Sugiyama, and T. Tani, Phys. Rev. B 66, 155103 (2002).

${ }^{28}$ D. J. Singh, Phys. Rev. B 76, 085110 (2007).

${ }^{29}$ A. Rebola, R. Klie, P. Zapol, and S. Ogut, Phys. Rev. B 85, 155132 (2012).

${ }^{30}$ J. Soret and M.-B. Lepetit, Phys. Rev. B 85, 165145 (2012).

${ }^{31}$ Y. Saeed, N. Singh, and U. Schwingenschlögl, Adv. Funct. Mater. 22, $2792(2012)$

${ }^{32}$ S. Lemal, J. Varignon, D. I. Bilc, and P. Ghosez, Phys. Rev. B 95, 075205 (2017).

${ }^{33}$ D. J. Singh, Mater. Res. Soc. Symp. Proc. 1044, 1044-U02-05 (2007).

${ }^{34}$ H. Ohta, K. Sugiura, and K. Koumoto, Inorg. Chem. 47, 8429 (2008).

${ }^{35}$ J. He and Y. Liu, J. Mater. Res. 26, 1762 (2011).

${ }^{36}$ S. Altin, M. A. Aksan, and A. Bayri, J. Alloys Compd. 587, 40 (2014).

${ }^{37}$ N. Karaman, A. Bayri, and S. Ekmekçi, J. Phys.: Conf. Ser. 667, 012008 (2016).

${ }^{38}$ G. Kresse and J. Hafner, Phys. Rev. B 47, 558 (1993).

${ }^{39}$ J. P. Perdew, K. Burke, and M. Ernzerhof, Phys. Rev. Lett. 77, 3865 (1996).

${ }^{40}$ P. Blaha, K. Schwarz, G. Madsen, D. Kvasicka, and J. Luitz, WIEN2k, An augmented plane wave plus local orbitals program for calculating crystal properties, TU Vienna, Vienna, 2001.

${ }^{41}$ G. K. H. Madsen, K. Schwarz, P. Blaha, and D. J. Singh, Phys. Rev. B 68, 125212 (2003).

${ }^{42}$ G. K. H. Madsen and D. J. Singh, Comput. Phys. Commun. 175, 67 (2006).

${ }^{43}$ K. P. Ong, D. J. Singh, and P. Wu, Phys. Rev. Lett. 104, 176601 (2010).

${ }^{44}$ M. E. Gruner, U. Eckern, and R. Pentcheva, Phys. Rev. B 92, 235140 (2015). 